Volume 8. No. 10, October 2020

International Journal of Emerging Trends in Engineering Research

Available Online at http://www.warse.org/IJETER/static/pdf/file/ijeter228102020.pdf

https://doi.org/10.30534/ijeter/2020/228102020

\title{
Development of a Risk Management algorithm in the Chemical industry
}

\author{
Olga Berezhnaya $^{1^{*}}$, Alexey Tolmachev ${ }^{1}$, Elena Berezhnaya ${ }^{1}$, Natalia Solovyova ${ }^{2}$, Marina Churakova ${ }^{3}$ \\ ${ }^{1}$ North Caucasian Federal University, Russia, ivanovpetr090820@gmail.com \\ ${ }^{2}$ Nevinnomyssk Institute of Economics, Management and Law, Russia; \\ ${ }^{3}$ Armavir Institute of Mechanics and Technology (branch of Kuban State Technological University), Russia
}

\begin{abstract}
The specificity of chemical production and potential environmental threats from chemical enterprises' activities have led to the relevance of research in the field of risk management. This circumstance forces us to actively use risk management tools to ensure the security of enterprises. At the same time, special attention is paid to risk management of uncontrolled environmental factors. Mathematical models are proposed for probabilistic analysis of the reliability of the system of a chemical enterprise. The main stages of modeling are selected, and the difficulties of implementing each step are analyzed. Methods of transformation and schemes of using the technology of structural and logical modeling for calculating the system's reliability are proposed.
\end{abstract}

Key words: modeling systems, investment risks, risk management, management model.

\section{INTRODUCTION}

Chemical industry enterprises are subject to a large number of risks, primarily due to the specifics of production. Also, due to the rapid development of production technologies, changes in prices for raw materials, there is a high competitive risk in relation to the products of chemical enterprises.

In addition, taking into account the complexity of production, the specifics of the raw materials used and the peculiarities of the organization of modern enterprises in the chemical industry, risk management is a necessity, since it is aimed at predicting potential threats, promptly responding to them. The risk management system allows you to quickly adapt to negative changes in the external environment and prevent such serious consequences of the influence of external factors as production shutdowns, accidents, etc.

To achieve the efficiency of enterprise risk management, an integrated risk management system is needed, which consists in an integrated approach to assessing the maximum number of risk factors for an enterprise's activities carried out in a dynamic economic environment. In order to obtain a holistic view of the risks inherent in chemical enterprises, we systematize the data obtained on the risk factors of the enterprises under consideration.

Russia fully possesses the necessary fundamental factors to ensure competitiveness in the world market. However, the analysis of the positions of the Russian chemical complex in the world shows that the potential formed by these factors is not fully utilized due to the presence of systemic problems in the industry. Taking into account the relevance and importance of this sector of the economy, a number of key problems of the chemical industry of the Russian Federation can be identified:

1) high prices and lack of the required range of raw materials for the chemical industry;

2) high level of wear and tear of a number of production facilities;

3) high capital costs for the construction of new chemical plants;

4) insufficient development of the scientific and technological potential of the chemical complex;

5) high prices for electricity and rail transportation;

6) insufficient development of standards and quality control systems for products of the chemical complex;

7) insufficient capacity of the domestic market;

8) dependence of strategic industries on imported raw materials;

9) insufficient development of human resources and high-performance jobs.

In this regard, the question arises about the quality of research, during which the risk assessment is carried out. It is clear that in order to calculate probabilistic indicators, it is necessary to build a mathematical (probabilistic) model that allows calculating the frequency of occurrence of an undesirable event, the likelihood of a hazardous chemical leaving the equipment, unavailability coefficients of the safety system, etc.

\section{MATERIALS AND METHODS}

Risk analysis is considered as a structured process, the purpose of which is to determine the likelihood and extent of adverse consequences of the investigated action, object or system. Harm to people, property or the environment is 
considered as adverse consequences.

As a result of the analysis, answers should be obtained to three main questions:

- What can fail (hazard identification)? - How likely is this to happen (frequency analysis)?

- What are the consequences of this event (analysis of consequences)?

Of great interest in risk analysis is the process of obtaining a probabilistic risk model that allows calculating the frequency of occurrence of an undesirable event, the likelihood of a technological process going beyond acceptable limits, coefficients of unavailability of the safety system, etc. Among the probabilistic and theoretical methods of quantitative risk analysis, the following methods can be distinguished: statistical methods, simulation modeling (Monte Carlo simulation method, historical simulation method), tree construction methods (event trees, fault trees, cause-effect), logical and probabilistic methods. The last two methods are of particular interest. Whichever method is chosen, when analyzing real complex systems, it is practically impossible to directly obtain the required calculated expressions. Therefore, the desired probabilistic model is usually obtained in several stages.

\section{RESULTS AND DISCUSSION}

At the first stage, a verbal description of the critical conditions of the system functioning leading to an accident occurs - a verbal safety model is developed. At the second stage, the verbal model is formalized, i.e. described using one or another mathematical apparatus.

At the third stage, the graphical model is transformed into a model represented by means of logic algebra (mathematical logic)

- logical function. Logical functions usually represent either a set of minimum flow areas (MAS), or - emergency combinations (MPS). MAS is such a conjunction of negations of its elements, none of the components of which can be removed without violating the conditions of system inoperability. This conjunction can be written as the following logical function (1):

$$
S_{j}=\hat{i}_{i \in K_{S_{j}}} x_{i}
$$

where $\mathrm{K}_{\mathrm{Sj}}$ - set of numbers corresponding to a given combination, xi - system elements.

MPS is such a conjunction of its elements, none of which can be removed without disrupting the functioning of the system. Such a conjunction can be written as follows (2):

$$
\mathscr{P}_{l}=\hat{i \in K}_{\mathscr{Q}_{l}} x_{i}
$$

where $\mathrm{K}_{\mathrm{Pl}}$ - set of numbers corresponding to a given section.

At the fourth stage, the logical model is transformed into a calculated probabilistic function, presented either in perfect disjunctive normal form, or in repetitive disjunctive normal form, or in orthogonal disjunctive normal form. This stage is the most time consuming. There are many methods for moving from a logical model to a probabilistic function. So, according to the tabular method and MAS, the probability of failure-free operation of the system can be obtained by the formula (3):

$$
\begin{gathered}
P\left\{y\left(x_{1}, \ldots, x_{n}\right)=1\right\}=R_{c}=P\left\{\begin{array}{l}
d \\
\underset{l=1}{\vee} \mathcal{P}_{i}
\end{array}\right\}=\sum_{i} P\left(P_{i}\right)-\sum_{j} \sum_{i} P\left(P_{i} \wedge \mathscr{P}_{j}\right)+ \\
+\sum_{k} \sum_{j} \sum_{i} P\left(\mathscr{P}_{i} \wedge \mathscr{P}_{j} \wedge \mathscr{P}_{k}\right)-\ldots+(-1)^{d-1} P\left(\mathscr{P}_{1} \wedge \mathscr{P}_{2} \wedge \ldots \wedge \mathscr{P}_{d}\right) .
\end{gathered}
$$

At the fifth stage, based on the probabilistic function polynomial, the required probabilistic safety characteristics are calculated, the model is checked and, if necessary, adjusted.

At the stage of building a graphical model, the main part of the regularities of the enterprise's functioning is displayed. The quality of the graph construction largely determines the adequacy of the model and the accuracy of subsequent calculations. This determines the exceptional importance of this stage.

Quite important and the most laborious is the stage of obtaining the polynomial probability function. Currently, there are a large number of software tools that automate the process of obtaining the IMF. At the same time, in the "fault tree" technology, an approximate probability function is used (often "insignificant" MAS are cut off), which are built on the basis of a logical function in the form of MAS, and in the technology of the general logical-probabilistic method, the logical function is transformed according to special rules into an exact probabilistic polynomial. However, the process of moving from the function of operability (inoperability) of the system to the IMF is laborious using any technology. Therefore, it is necessary to look for new ways to obtain the probability of an accident, based on unconventional methods, such as: artificial intelligence systems (neural networks), modeling based on the fuzzy logic apparatus, which are now widely used. Example. As an example, consider the functional diagram of the bridge structure (Figure 1). The probability of initiating events: $\mathrm{p}_{\mathrm{i}}=0.9, \mathrm{q}_{\mathrm{i}}=0.1$. 


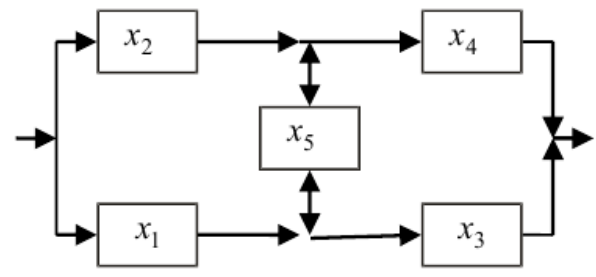

Figure 1: Functional diagram of the bridge structure

\section{CONCLUSION}

Effective risk management should be based on complete and reliable information, the structure and content of which satisfy the conditions of minimality and sufficiency for making management decisions. In addition, the information obtained must be properly systematized, interpreted, which implies the use of risk indicators for these purposes. Risk indicators are indicators that indicate the presence of certain threats to the activities of the enterprise.

The following requirements for operational risk indicators are proposed:

- risk indicators should be informative and should cover the whole variety of operations of an economic entity;

- risk indicators should be provided with reliable information necessary for their calculation;

- when setting normative values for risk indicators, it is necessary to comprehensively analyze the specifics of the enterprise and the current conditions of its functioning;

- the calculation of indicators should be carried out on an ongoing basis using operational relevant information.

When choosing risk indicators, the following problem arises, which economic indicators indicate risks to business reputation, for example, risks of losing key personnel, etc., risks that are more subjectively assessed based on certain facts.

Thus, the application of the risk management mechanism at the chemical industry enterprises will reduce the level of integral risk, as well as reduce the uncertainty of activities. In addition, the main focus of the risk management mechanism is to promptly identify and respond to emerging risk signals.

\section{REFERENCES}

1. Ya. D. Vishnyakov, A. V. Kolosov, V. L. Shemyakin (2000). Assessment and Analysis of Financial Risks of an Enterprise in an A priori Hostile Business Environment, Management in Russia and Abroad, Vol. 3, pp. 106-111.

2. R. M. Kachalov (2002). Business risk management. Moscow, Publishing house "Science", 192 p.

3. T. V. Polteva, N. V. Mingalev (2013). Analysis of financial investment instruments: the ratio of risk and return, Karelian Scientific Journal, Vol. 4, pp. 33-36.

4. T. V. Kramin, V. A. Leonov, A. V. Timiryasova (2013). Investment attractiveness of the region as the basis for the development and implementation of a regional investment project, Science Vector of Togliatti State University, 2 (24), 293-300.

5. A. A. Agapov, E. A. Agapova (2015). Software Certification and Verification, Industrial Safety, Vol. 4, 58-60.

6. S. Shin, S-H. Byeon, J-R. Sohn and K.W. Moon (2019). Development and Verification of a Risk Index for Evaluating the Chemical Accident Risk of Korean Chemical Enterprises, International Journal of Environmental Research and Public Health, Vol. 16, pp. 4409

7. S. Girgin, A. Necci, E. Krausmann (2019). Dealing with cascading multi-hazard risks in national risk assessment: The case of Natech accidents, International Journal of Disaster Risk Reduction, Vol. 35, 101072.

8. O. V. Sycheva, S. N. Shlykov, R. S. Omarov, and E. A. Skorbina (2019). Studying effect of electromagnetic treatment on milk technological properties, Engineering for Rural Development Proceedings, pp. 430-435.

9. O. V. Takhumova, T. I. Bondarenko, S. N. Shlykov, and Omarov R. S. (2019). Studying identification algorithms in food technology, Engineering for Rural Development Proceedings, pp. 1186-1191.

10. A. Pozmogov, R. Omarov, S. Shlykov, L. Badova, A. Tokaeva (2020). App Development for Enterprises Financial Insolvency Diagnostic with Legal Support, International Journal of Emerging Trends in Engineering Research, Vol. 8(9), pp. 5952-5955.

11. R. Omarov, I. Gorlov, M. Slozhenkina, N. Mosolova, S. Shlykov (2019). Applying methods of mathematical modeling in cattle breeding, International Journal of Innovative Technology and Exploring Engineering, 8(12), pp. 185-187.

12. A. D. M. Africa, A. M. S. Alejo, G. L. M. Bulaong, S. M. R. Santos, J. S. K. Uy (2020). Computer Vision on a Parking Management and Vehicle Inventory System, International Journal of Emerging Trends in Engineering Research, 8(2), pp. 323-332. 